\section{SUMMARY}

\section{P. P. Yablonsky, S. M. Yashin}

The first experience of orthotropic implantation of decellularized mitral allograft

Traditional biological and mechanical valve substitutes have some well-known limitation, such as rapid deterioration of the tissue ones in young patient and the high risk of thrombosis and anticoagulation therapy complications for the mechanical ones. At the same time the aortic and pulmonary valves can already be replaced with decellularized allografts that showed promising results in terms of both hemodynamics and reliability while anticoagulation for them is not needed. This paper describes the first orthotropic implantation of the decellularized mitral valve allograft in sheep model. The original method without stabilizing ring is described, which have shown good echocardiographic results.

Keywords: cardio-vascular surgery, mitral valve replacement, decellularization, mitral allograft.
(ㄷ) Коллектив авторов, 2015 г.

УАК 616.155.392.8-036.11-08:616.832-089.844

С. Н. Бондаренко, И. С. Моисеев,

И. А. Самородова, Т. Л. Гиндина,

М. А. Кучер, О. А. Слесарчук,

Л. С. Зубаровская, Б. В. Афанасьев

СРАВНЕНИЕ ЭФФЕКТИВНОСТИ ХИМИОТЕРАПИИ И АЛЛОГЕННОЙ ТРАНСПЛАНТАЦИИ ГЕМОПОЭТИЧЕСКИХ СТВОЛОВЫХ КЛЕТОК ПРИ ОСТРОМ МИЕЛОБЛАСТНОМ ЛЕЙКОЗЕ В ПЕРВОЙ РЕМИССИИ У ВЗРОСЛЫХ

НИИ детской онкологии, гематологии и трансплантологии имени Р. М. Горбачевой; кафедра гематологии, трансфузиологии и трансплантологии ФПО Первого Санкт-Петербургского государственного медицинского университета имени академика И. П. Павлова

\section{ВВЕДЕНИЕ}

ОМл - клональное заболевание системы крови, характеризующееся нарушением дифференцировки и неконтролируемой пролиферацией опухолевых клеток, вызывающей угнетение нормального гемопоэза. Аиагноз ОМЛ объединяет несколько вариантов заболевания, различных по клиническим проявлениям, морфологическим, иммунологическим и молекулярно-генетическим характеристикам, что определяет ответ на терапию и прогноз течения. Встречаемость ОМЛ колеблется в зависимости от возраста - дети до 19 лет - $6 \%$, от 20 до 60 лет - 33 \%, пациенты старше 60 лет - 61 \%. Возраст является одним из наиважнейших факторов прогноза [1]. Современная классификация ВОЗ (2008) основывается на цитогенетических и молекулярно-генетических аномалиях, которые лежат в основе прогноза заболевания [2]. Несмотря на большое количество исследований влияния кариотипа на результаты терапии, механизм лекарственной устойчивости при той или иной аномалии до сих пор изучен плохо [3]. Ввиду этого, непрерывно идет поиск факторов, ассоциированных с успехом при проведении ХТ и изучение роли и места аллоТГСК в терапии данной категории больных.

Индукционная ХТ, состоящая из одного или двух курсов цитарабина в сочетании с антрациклином, приводит в достижению полной ремиссии примерно у 80 \% пациентов в возрасте до 65 лет [4, 5]. ОМ характеризуется высокой частотой рецидивов заболевания у взрослых, долгосрочная безрецидивная выживаемость не превышает 45 \% [6, 7]. Наиболее эфрективным методом терапии ОМ $\curlywedge$ является аллоТГСК, как за счет цитостатического воздействия на лейкозные клоногенные клетки режимом кондиционирования, так и иммуноадоптивным эффектом «трансплантат против лейкоза» за счет донорских Т-лимфоцитов [8-10]. Однако, возможно развитие реакции «трансплантат против хозяина» (РТПХ) тяжелой степени из-за чрезмерной аллореактивности трансплантата, что может привести к летальному исходу. Показанием к проведению аллоТГСК в первой ремиссии при высокой цитогенетической группе риска подтверждены многими исследованиями. Результаты аллоТГСК в других цитогенетических группах риска не столь однозначны [11 - 13].

Целью исследования явилось сравнить эффрективность аллоТГСК и ХТ и выявить факторы, влияющие на долгосрочную выживаемость у пациентов ОМЛ в первой ремиссии.

\section{МАТЕРИАЛ И МЕТОДЫ ИССЛЕДОВАНИЯ}

В исследование включено 140 пациентов в возрасте 18 - 65 лет с диагнозом ОМ (кроме ОМл М3 FAB) в первой ремиссии, которым проводилась ХТ или аллоТГСК, при наличии НLA-совместимого донора (родственного 19 (27 \%) или неродственного 51 (73 \%)) в период 2005 по 2014 гг. Продолжительность наблюдения составила от 1 мес. до 99 мес. (медиана 25 мес.).

Распределились пациенты в соответствии с цитогенетическими группами риска: низкий риск (НР) (t(8;21) (q22;q22), inv16(p13.1;q22) у 20 (14\%) пациентов; стандартный риск (СР) (нормальный кариотип, хромосомные аномалии, не относящиеся к низкой 
и высокой группе риска) - 74 (53 \%) пациент; высокий риск (BP) (abn(3q), t(6;9) (p23;q34), abn(11q23), $\operatorname{del}(5 q) /-5, \operatorname{del}(7 q) /-7, \operatorname{abn}(17 p)$, комплексные аномалии кариотипа ( $\geq 3$ поломок)) - 41 (29\%). Цитогенетическое исследование не проводилось у 5 (4 \%) пациентов.

ХТ включала 2 курса индукции («7+3»), 2 курса консолидации («НАМ») и поддерживающая терапия ротирующими курсами в течение 1 года с интервалом в 4 недели («5+2»). Пациентам, которым не была достигнута полная ремиссия после первого курса, проводился второй курс индукции по схеме «НАМ», таких насчитывалось 46 (33\%). Не удалось достичь полной ремиссии после 2-х курсов индукционной терапии у 18 (13\%) пациентов, в последующем они не включались в сравнительный анализ.

Всем пациентам цитогенетических СР и ВР групп, которым были найдены HLA-совместимые доноры как родственные, так и неродственные, проводилась аллоТГСК. Показанием к проведению аллоТГСК в первой ремиссии пациентам низкой группы риска являлись: исходный гиперлейкоцитоз $\left(>20,0 \times 10^{9} / \lambda\right)$, отсутствие полной ремиссии после первого курса индукции и наличие признаков минимальной резидуальной болезни после окончания курсов консолидации.

При аллоТГСК использовались миелоаблативный режим кондиционирования у 18 (26 \%) пациентов, включающий бусульфан 16 мг/кг и циклофоссран 120 мг/кг, а также режим кондиционирования со сниженной интенсивностью - 52 (74\%), включающий флюдарабин 150 - 180 мг/м² и бусульфан 8 мг/кг или мелфалан 140 мг/м².

Режимы кондиционирования со сниженной интенсивностью назначали пациентам старше 40 лет, а также с высоким индексом коморбидности и имевшим тяжелые осложнения при проведении индукционной и консолидационной химиотерапии.

В качестве профилактики острой РТПХ использовали циклоспорин А или такролимус в комбинации с метотрексатом или мофетила микофенолатом. При аллоТГСК от неродственного донора добавляли антилимфоцитарный глобулин.

Сравнительная характеристика пациентов представлена в табл. 1.

Анализ ОВ, БСВ, РР и летальность, несвязанную с заболеванием (ЛНЗ) проводили по методу Каплан-Майера, используя log-rank тест для оценки достоверности различий и пропорциональную модель Кокса с определением значения отношения рисков и его $95 \%$ доверительного интервала (HR(CI95\%)). Отправной точкой отсчета считалась дата достижение полной ремиссии заболевания, конченой точкой - дата последнего контакта для живых пациентов и дата смерти при анализе ОВ, и дата рецидива или дата смерти в ремиссии на фоне
Таблица 1

Сравнительные характеристики пациентов

\begin{tabular}{|c|c|c|}
\hline Характеристика & аялоТГС & XT \\
\hline Количество & 70 & 70 \\
\hline Возраст (медиана), лет & 18-62 (37) & $18-65(45)$ \\
\hline $\begin{array}{l}\text { Алительность наблюдения } \\
\text { (медиана), мес. }\end{array}$ & 5-99 (29) & $1-78(21)$ \\
\hline $\begin{array}{l}\text { Пол, n (\%) } \\
\text { мужчины } \\
\text { женщины } \\
\end{array}$ & $\begin{array}{l}22(31) \\
48(69)\end{array}$ & $\begin{array}{l}32(46) \\
38(54)\end{array}$ \\
\hline $\begin{array}{l}\text { FAВ классификация, n (\%) } \\
\text { M0 } \\
\text { M1 } \\
\text { M2 } \\
\text { M4 } \\
\text { M5 } \\
\text { M6 } \\
\text { M7 }\end{array}$ & $\begin{array}{l}10(14) \\
14(20) \\
14(20) \\
15(21) \\
11(16) \\
5(7,5) \\
1(1,5)\end{array}$ & $\begin{array}{c}7(10) \\
15(21) \\
19(27) \\
17(24) \\
9(13,5) \\
2(3) \\
1(1,5)\end{array}$ \\
\hline $\begin{array}{l}\text { Происхождение ОМ } \\
\text { de nоvo } \\
\text { вторичный }\end{array}$ & $\begin{array}{l}58(83) \\
12(17)\end{array}$ & $\begin{array}{l}56(80) \\
14(20)\end{array}$ \\
\hline $\begin{array}{l}\text { Лейкоциты, } \times 109 / \wedge, \mathrm{n}(\%) \\
\leq 20,0 \\
21,0-100,0 \\
>100,0\end{array}$ & $\begin{array}{l}37(52) \\
22(32) \\
11(16) \\
\end{array}$ & $\begin{array}{l}41(59) \\
21(30) \\
8(11) \\
\end{array}$ \\
\hline $\begin{array}{l}\text { Цитогенетическая } \\
\text { классификация, n (\%) } \\
\text { низкий } \\
\text { стандартный } \\
\text { высокий } \\
\text { не известно } \\
\end{array}$ & $\begin{array}{l}8(11) \\
40(57) \\
22(32) \\
\quad- \\
\end{array}$ & $\begin{array}{c}12(17) \\
34(49) \\
19(27) \\
5(7)\end{array}$ \\
\hline $\begin{array}{l}\text { Курсов индукции до полной } \\
\text { ремиссии, } \mathrm{n}(\%) \\
1 \\
2 \\
\text { не достигнута }\end{array}$ & $\begin{array}{l}43(61) \\
27 \\
\quad-\end{array}$ & $\begin{array}{l}33(47) \\
19(27) \\
18(26)\end{array}$ \\
\hline
\end{tabular}

терапии или дата последнего контакта для живых в первой полной ремиссии пациентов при анализе БСВ. Проводился анализ конкурирующих рисков РР и АНЗ. Многофакторный анализ проводили методом пропорциональных интенсивностей Кокса. Статистически достоверными считали различия при р®,05. Обработку результатов выполняли в программе SPSS Statistics v.22 и Easy R.

\section{РЕЗУЛЬТАТЫ ИССЛЕДОВАНИЯ И ИХ ОБСУЖДЕНИЕ}

На момент окончания исследования в группе аллоТГСК живы 49 (70\%) пациентов, а при ХТ - 24 (46 \%) пациента. Полный сравнительный однофакторный анализ пациентов двух групп представлен в табл. 2.

В течение 5-ти лет РР значительно меньше в группе аллоТГСК (24\% против 57 \%, соответственно, HR,0,36(CI95\%0,19-0,7) p=0,003) (рис. 1).

В СР и ВР цитогенетической группах Аля пациентов после аллоТГСК РР отмечался статистически достоверно меньше (HR,0,2(CI95 \%0,07-0,56) $\mathrm{p}=0,002$ и НR, 0,27 (CI95 \%0,08-0,86) p=0,03, соответственно) (рис. 2; 3), тогда как в группе цитогенетического НР РР оказался сопоставим (HR, 1,33(CI95\% 0,26-6,6) p=0,7). РР был меньше после аллоТГСК не зависимо от возраста (моложе и старше 40 лет) $(\mathrm{HR}, 0,33(\mathrm{CI} 95 \% 0,13-0,81) \mathrm{p}=0,02$ и HR,0,38(CI95\% $0,14-1,0) \mathrm{p}=0,05$, соответственно). 
Таблица 2

\begin{tabular}{|c|c|c|c|c|c|c|c|c|}
\hline & \multicolumn{3}{|c|}{ аллоТГС } & \multicolumn{3}{|c|}{$\mathrm{XT}$} & \multirow{2}{*}{$\mathrm{p}$} & \multirow{2}{*}{ HR (CI 95 \%) } \\
\hline & $\mathrm{n}$ & $\mathrm{n}$ & $5-\mathrm{B} \pm \mathrm{CO} 1, \%$ & $\mathrm{n}$ & $\mathrm{n}$ & $5-\mathrm{B} \pm \mathrm{CO} 1, \%$ & & \\
\hline Все пациенты & 70 & & & 52 & & & & \\
\hline $\mathrm{OB}^{2}$ & & 52 & $67 \pm 6$ & & 30 & $46 \pm 5$ & 0,02 & $0,48(0,26-0,9)$ \\
\hline БCB $^{3}$ & & 21 & $65 \pm 6$ & & 28 & $30 \pm 4$ & 0,001 & $0,39(0,22-0,69)$ \\
\hline $\mathrm{PP}^{4}$ & & 15 & $24 \pm 5$ & & 22 & $57 \pm 10$ & 0,003 & $0,36(0,19-0,7)$ \\
\hline \multicolumn{9}{|c|}{ Возраст } \\
\hline Ао 40 лет & 44 & & & 22 & & & & \\
\hline $\mathrm{OB}^{2}$ & & 34 & $74 \pm 6$ & & 14 & $51 \pm 6$ & 0,2 & $0,54(0,21-1,36)$ \\
\hline $\mathrm{5CB}^{3}$ & & 13 & $68 \pm 7$ & & 12 & $18 \pm 5$ & 0,02 & $0,4(0,18-0,87)$ \\
\hline $\mathrm{PP}^{4}$ & & 9 & $22 \pm 6$ & & 10 & $70 \pm 11$ & 0,02 & $0,33(0,13-0,81)$ \\
\hline Старше 40 лет & 26 & & & 30 & & & & \\
\hline $\mathrm{OB}^{2}$ & & 18 & $56 \pm 7$ & & 16 & $44 \pm 7$ & 0,1 & $0,48(0,2-1,13)$ \\
\hline БС $\mathrm{CB}^{3}$ & & 8 & $60 \pm 7$ & & 16 & $38 \pm 7$ & 0,03 & $0,39(0,17-0,92)$ \\
\hline $\mathrm{PP}^{4}$ & & 6 & $27 \pm 5$ & & 12 & $48 \pm 9$ & 0,05 & $0,38(0,14-1,0)$ \\
\hline \multicolumn{9}{|c|}{ Острый миелобластный лейкоз } \\
\hline De novo & 58 & & & 46 & & & & \\
\hline $\mathrm{OB}^{2}$ & & 44 & $66 \pm 7$ & & 28 & $48 \pm 5$ & 0,07 & $0,52(0,26-1,05)$ \\
\hline $\mathrm{БCB}^{3}$ & & 17 & $63 \pm 7$ & & 23 & $36 \pm 5$ & 0,009 & $0,44(0,23-0,81)$ \\
\hline $\mathrm{PP}^{4}$ & & 11 & $22 \pm 5$ & & 17 & $49 \pm 8$ & 0,01 & $0,37(0,17-0,79)$ \\
\hline Вторичный & 12 & & & 6 & & & & \\
\hline $\mathrm{OB}^{2}$ & & 8 & $67 \pm 10$ & & 2 & $33 \pm 14$ & 0,06 & $0,28(0,07-1,13)$ \\
\hline $\mathrm{5CB}^{3}$ & & 4 & $67 \pm 11$ & & 5 & $17 \pm 12$ & 0,08 & $0,31(0,8-1,18)$ \\
\hline $\mathrm{PP}^{4}$ & & 4 & $33 \pm 12$ & & 5 & $83 \pm 7$ & 0,08 & $0,31(0,8-1,18)$ \\
\hline \multicolumn{9}{|c|}{ Первичная резистентность } \\
\hline$\Delta \mathrm{a}$ & 27 & & & 19 & & & & \\
\hline $\mathrm{OB}^{2}$ & & 15 & $40 \pm 4$ & & 10 & $42 \pm 7$ & 0,5 & $0,86(0,56-1,33)$ \\
\hline $\mathrm{5CB}^{3}$ & & 13 & $39 \pm 4$ & & 11 & $28 \pm 7$ & 0,1 & $0,74(0,49-1,11)$ \\
\hline $\mathrm{PP}^{4}$ & & 9 & $40 \pm 8$ & & 9 & $59 \pm 11$ & 0,1 & $0,68(0,43-1,08)$ \\
\hline Нет & 43 & & & 33 & & & & \\
\hline $\mathrm{OB}^{2}$ & & 37 & $85 \pm 5$ & & 20 & $49 \pm 7$ & 0,006 & $0,53(0,33-0,86)$ \\
\hline $\mathrm{БCB}^{3}$ & & 8 & $80 \pm 6$ & & 17 & $30 \pm 6$ & 0,001 & $0,52(0,34-0,79)$ \\
\hline $\mathrm{PP}^{4}$ & & 6 & $15 \pm 5$ & & 13 & $56 \pm 11$ & 0,006 & $0,51(0,31-0,82)$ \\
\hline \multicolumn{9}{|c|}{ Цитогенетическая группа риска } \\
\hline Низкая & 8 & & & 11 & & & & \\
\hline $\mathrm{OB}^{2}$ & & 5 & $63 \pm 14$ & & 9 & $74 \pm 7$ & 0,5 & $1,89(0,32-11,38)$ \\
\hline $\mathrm{БCB}^{3}$ & & 3 & $60 \pm 13$ & & 4 & $55 \pm 8$ & 0,9 & $0,98(0,22-4,41)$ \\
\hline $\mathrm{PP}^{4}$ & & 3 & $37 \pm 15$ & & 3 & $31 \pm 12$ & 0,7 & $1,33(0,26-6,6)$ \\
\hline Стандартная & 40 & & & 28 & & & & \\
\hline $\mathrm{OB}^{2}$ & & 33 & $79 \pm 6$ & & 17 & $52 \pm 7$ & 0,03 & $0,35(0,14-0,91)$ \\
\hline БС $\mathrm{B}^{3}$ & & 8 & $78 \pm 6$ & & 15 & $29 \pm 7$ & 0,001 & $0,26(0,11-0,61)$ \\
\hline $\mathrm{PP}^{4}$ & & 5 & $14 \pm 6$ & & 12 & $60 \pm 11$ & 0,002 & $0,2(0,07-0,56)$ \\
\hline Высокая & 22 & & & 9 & & & & \\
\hline $\mathrm{OB}^{2}$ & & 14 & $38 \pm 9$ & & 2 & $19 \pm 5$ & 0,005 & $0,25(0,09-0,69)$ \\
\hline $5 \mathrm{CB}^{3}$ & & 10 & $34 \pm 10$ & & 7 & $17 \pm 3$ & 0,007 & $0,22(0,08-0,64)$ \\
\hline $\mathrm{PP}^{4}$ & & 7 & $37 \pm 11$ & & 5 & $76 \pm 12$ & 0,03 & $0,27(0,08-0,86)$ \\
\hline
\end{tabular}

П р и м е ч а н и е : 1 - пятилетняя выживаемость \pm стандартная ошибка; 2 - общая выживаемость; 3 - бессобытийная выживаемость; 4 - риск рецидива.

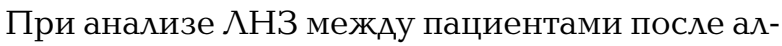
лоТГСК и ХТ не получено статистически достоверной разницы при сравнении по возрасту, уровню лейкоцитов, происхождению ОМ $\Lambda$, ответу на индукционную терапию и цитогенетическим группам риска.

В результате получена достоверно выше БСВ у пациентов после аллоТГСК по сравнению с ХТ (65\% против $30 \% \mathrm{HR}, 0,39(\mathrm{CI} 95 \% 0,22-0,69) \mathrm{p}=0,001)$ (рис. 4).

Улучшение БСВ наблюдалось в обеих возрастных группах (HR,0,4(CI95 \%0,18-0,87) $\mathrm{p}=0,02$ и $\mathrm{HR}, 0,39$ (CI95 \% 0,17-0,92) p =0,03, соответственно. БСВ была выше у пациентов с ОМ $\lambda$ de novo в сравнении с вторичным OM $\Lambda$ (HR, 0,44 (C) $95 \%$ $0,23-0,81) \mathrm{p}=0,009)$, у пациентов с достижением полной ремиссии после первого курса индукции $(\mathrm{HR}, 0,52(\mathrm{CI} 95 \% 0,24-0,79) \mathrm{p}=0,001)$, а также в CP и ВР цитогенетических группах (HR,0,26(CI95 \% $0,11-0,61) \mathrm{p}=0,001$ и $\mathrm{HR}, 0,25(\mathrm{CI} 95 \% 0,09-0,69)$ $\mathrm{p}=0,007$, соответственно).

Улучшение БСВ у пациентов после аллоТГСК отразилось в улучшении 5-летней ОВ по сравнению с пациентами в группе XT, но разница была менее 
ощутима (67 против $46 \%$, соответственно, HR,0,48 (СI95\%0,26-0,9) $\mathrm{p}=0,02)$ (рис. 5).

Повышение ОВ также наблюдалось у пациентов без первичной резистентности (HR,0,53(CI95\% $0,33-0,86) p=0,006)$ и в цитогенетических группах СР и ВP $(\mathrm{HR}, 0,35(\mathrm{CI} 95 \% 0,14-0,91) \mathrm{p}=0,03$ и $\mathrm{HR}, 0,22$ (СI95\%0,08-0,64) p =0,005, соответственно), тогда как у пациентов цитогенетической группы НР различий ОВ не наблюдалось (HR,1,89(СI95 \%0,32$11,38) \mathrm{p}=0,5)$.

При проведении многофакторного Кокс регрессионного анализа у пациентов ОМ $/$ в первой полной ремиссии выявлено, что на ОВ и БСВ влияет проведение алмоТГСК (HR,0,37(CI95 \%0,19-0,73) $\mathrm{p}=0,004$ и НR, $0,39(\mathrm{CI} 95 \% 0,21-0,72) \mathrm{p}=0,002$, соответственно) и отношение к цитогенетической группе риска: низкой (HR, 2,14(CI95 \% 1,24-3,71) $\mathrm{p}=0,006$ и НR, 1,93(CI95 \% 1,19-3,13) p =0,008, соответственно) и высокой $(\mathrm{HR}, 2,93$ (CI95 \% $1,45-5,9) \mathrm{p}=0,003$ и $\mathrm{HR}, 3,12$ (CI95 \% 1,62-6,0) $\mathrm{p}=0,001$, соответственно). Аля оценки влияния расширенного количества факторов: пола (женщины/мужчины), возраста (моложе и старше 40 лет), количества лейкоцитов при диагностике OM $\Lambda$ (20,0/21,0 - 100,0/>100,0× $\times 10^{9} /$ л) и первичная резистентность (да/нет), на ОВ,

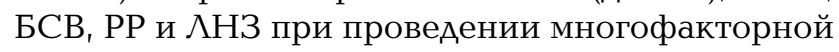
регрессии Кокса они были добавлены в модель. Из них отмечено негативное влияние первичной резистентности на ОВ, БСВ и $\mathrm{PP}(\mathrm{HR}, 1,9(\mathrm{CI} 95 \%$ $1,0-3,66) \mathrm{p}=0,05, \mathrm{HR}, 2,07(\mathrm{CI} 95 \% 1,15-3,72) \mathrm{p}=0,02$ и НR, 0, 47 (CI95 \%0,3-0,74) p=0,001, соответственно) и только уровень лейкоцитов (>20,0× $10^{9} /$ ^) повышал PP (HR, 1,91 (CI95\%1,09-3,32) p=0,02). Подробные данные многофакторного анализа представлены в табл. 3.

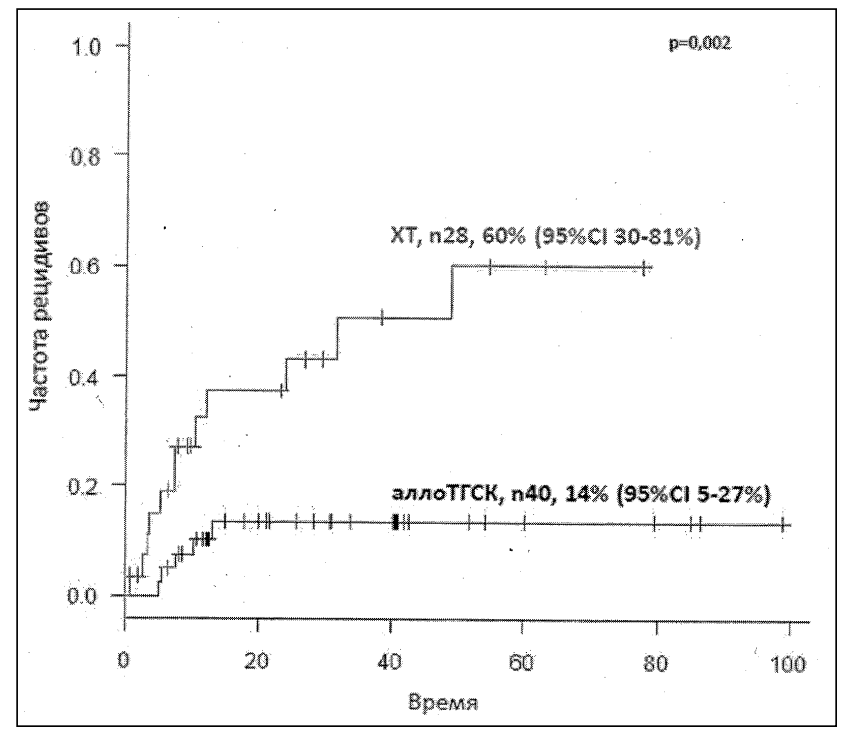

Рис. 2. Сравнение частоты рецидивов при ОМЛ в ПР1 после аллоТГСК и ХТ в зависимости от цитогенетической группы риска (стандартная)

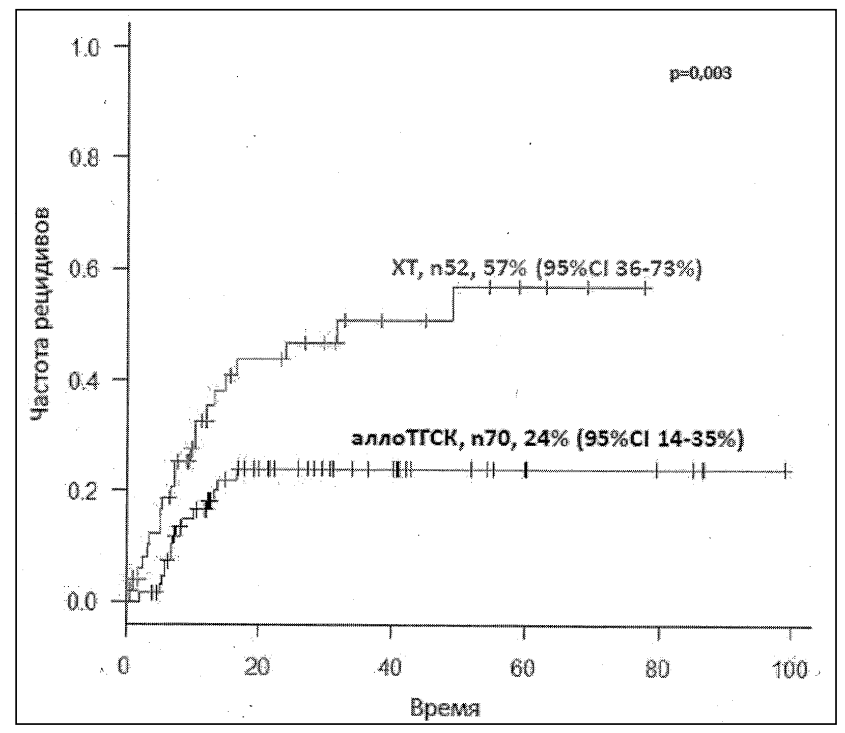

Рис. 1. Сравнение частоты рецидивов при ОМЛ в ПР1 после аляоТГСК и ХТ

По результатам нашего исследования показана лучшая 5-ти летняя ОВ (67 против $46 \%$ ), и особенно БСВ (65 против $30 \%$ ) (рис. $1 ; 2)$ у пациентов после аллоТГСК по сравнению с XТ, что подтверждается результатами ранее проведенных исследований EORCT/GIMEMA AML-10trail (58 против 50 \% и 52 против $42 \%$, соответственно) [10]. Это происходит за счет меньшей частоты рецидивов в группе пациентов после аллоТГСК (24 против 57 \%) (рис. 3), что обусловлено эффектом трансплантат-противлейкоза. ОАнако в нашем исследовании обращает на себя внимание сравнимая $\lambda Н 3$ у пациентов после аллоТГСК и ХТ (11 против $13 \%$ ), что отличается

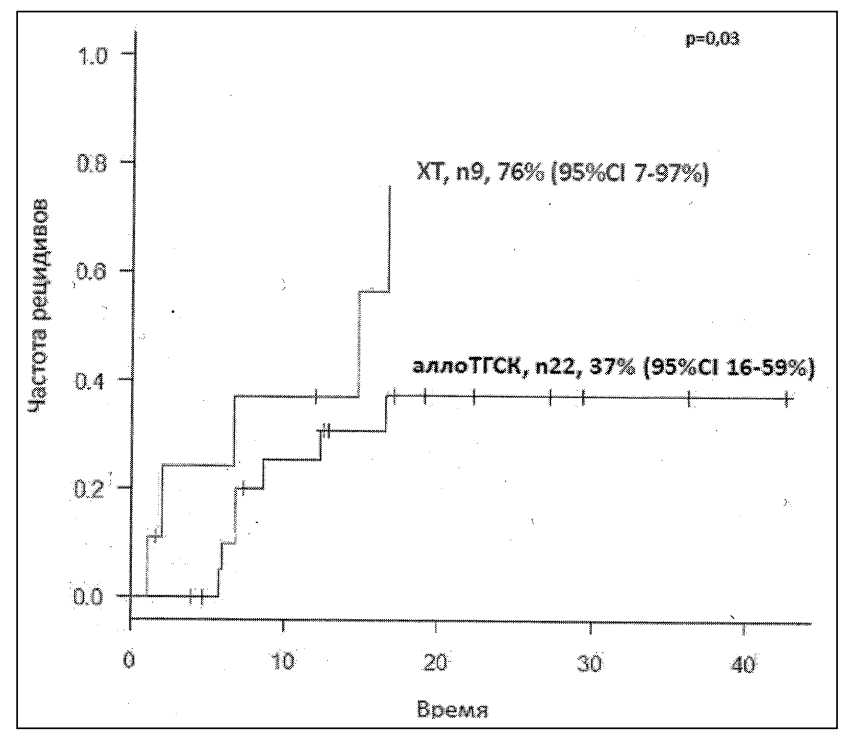

Рис. 3. Сравнение частоты рецидивов при ОМЛ в ПР1 после амлоТГСК и ХТ в зависимости от цитогенетической группы риска (высокая) 


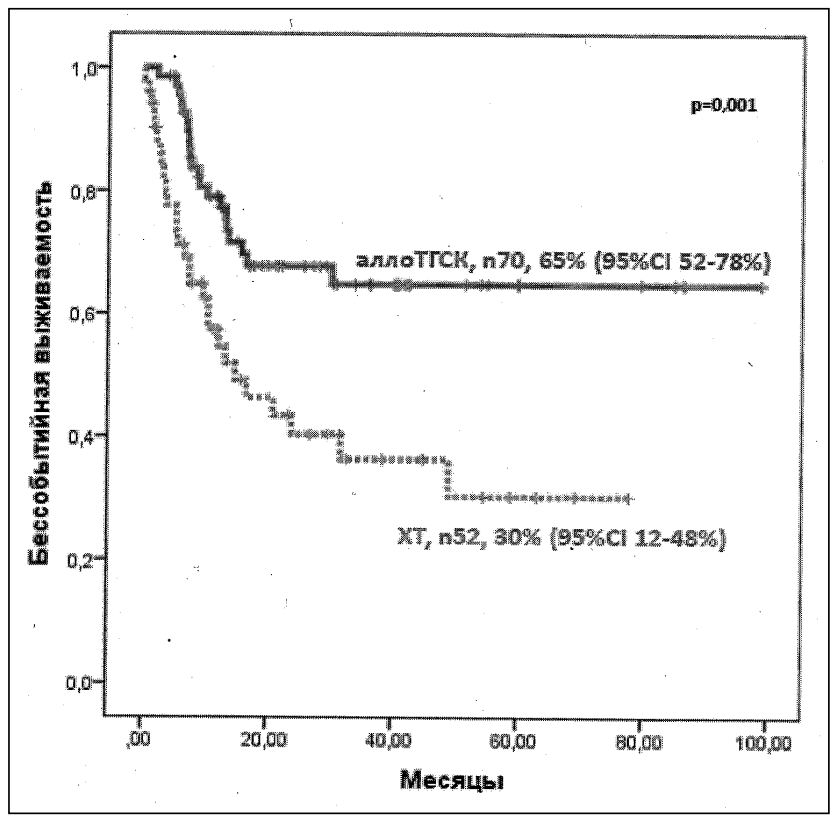

Рис. 4. Бессобытийная выживаемость при ОМ $\Lambda$ в ПР1 после аллоТГСК и ХТ

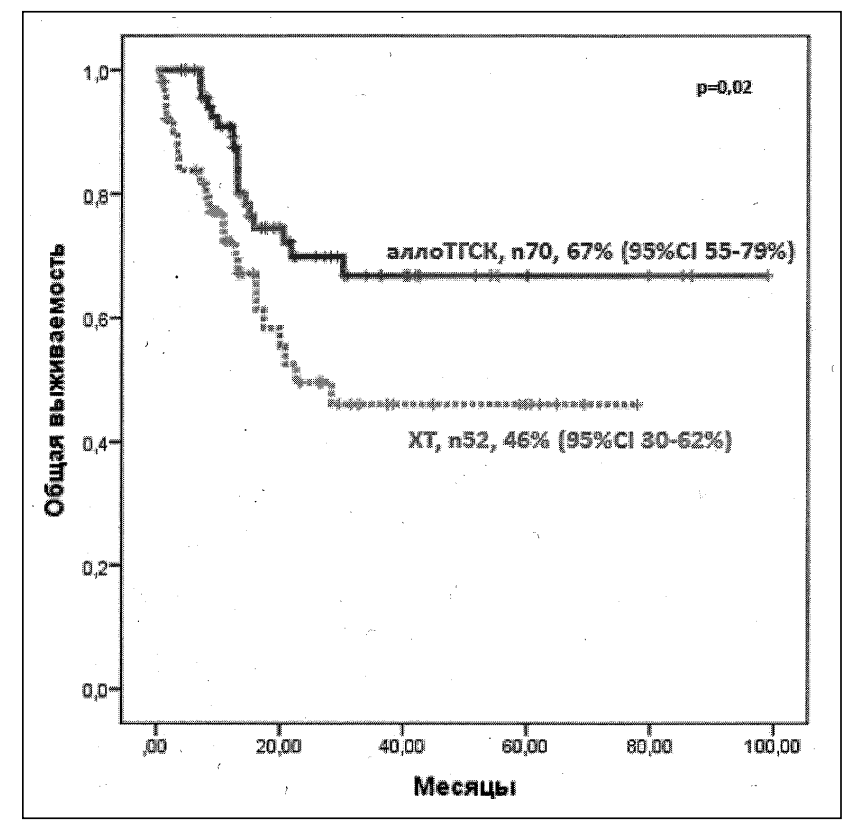

Рис. 5. Общая выживаемость при ОМ $\lambda$ в ПР1 после аллоТГСК и ХТ

соответственно), исследование HOVON/SAKK публикует схожие результаты (40 против $30 \%$, 33 против 17 \% и 39 против $77 \%$, соответственно) [12]. Высокая частота рецидивов (>35\%) у пациентов цитогенетической группы ВР, Ааже после аллоТГСК, вынуждает рассматривать новые подходы в раннем посттрансплантационном периоде, такие как назначение 5-азацитидина для дополнения иммуноадоптивного эффекта «трансплантатпротив-лейкоза»[13].

Помимо неблагоприятного влияния на ОВ и БСВ цитогенетических групп СР и ВР на основании многофакторного анализа, получен такой фактор риска, как отсутствие полной ремиссии после первого индукционного курса ХТ (табл. 3) [4]. Увеличивают частоту рецидивов также высокий уровень лейкоцитов в дебюте заболевания $\left(>21,0 \times 10^{9} /\right.$ л) и вторичный ОМ $\Lambda$ [4]. Эти факторы риска лишний раз подчеркивают важность аллоТГСК у данной категории пациентов, и необходимость HLAтипирования пациента до начала индукционной терапии, чтобы избежать задержки с проведениемаллоТГСК.

\section{ЗАКЛЮЧЕНИЕ}

Таким образом, эффективность аллоТГСК по сравнению с ХТ выше при цитогенетических группах СР и ВР. Аругие факторы такие, как происхождение OM $\wedge$ (de novo/вторичный), уровень лейкоцитов в дебюте заболевания и ответ на первый курс ХT, могут рассматриваться как дополнительные критерии в пользу аллоТГСК. В настоящее время ал^оТГСК у пациентов ОМЛ в цитогенетических 
групп СР и ВР является показанием для выполнения в первой ремиссии при наличии HLA-совместимого донора.

\section{ЛИТЕРАТУРА}

1. Dores G. M. et al. Acute leukemia incidence and patient survival among children and adults in the United States. $2001-$ 2007 // Blood. - 2012. - № 119 (1). P. $34-43$.

2. Swerdlow S. H. et al. WHO Classification of Tumours of Haematopoietic and Lymphoid Tissues: International Agency for Research on Cancer. $-4^{\text {th }}$ ed. - Lyon: IARC Press, 2008.

3. Dohner H. et al. Diagnosis and management of acute myeloid leukemia in adults: recommendations from an international expert panel, on behalf of the European Leukemia // Net. Blood. - 2010. - № 115. P. $453-474$

4. Parovichnikova E. N. et al. Treating patients with acute myeloid leukemias (AML) according to the protocol of the AML01.10 Russian multicenter randomized trial: the coordinating center's results // Ter. Arkh. - 2014. - № 86 (7). - P. 14-23.

5. Burnett A. K., Hills R. K., Milligan D. W. et al. Attempts to Optimize Induction and Consolidation Treatment in Acute Myeloid Leukemia: Results of the MRC AML12 Trial // J. Clin. Oncol. - 2010. - № 28. - P. 586-595.

6. Schaich M., Rollig C., Soucek S. et al. Cytarabine dose of 36 $\mathrm{g} / \mathrm{m} 2$ compared with $12 \mathrm{~g} / \mathrm{m} 2$ within first consolidation in acute myeloid leukemia: results of patients enrolled onto the prospective randomized AML96 study // J. Clin. Oncol. - 2011. № 29. - P. $2696-2702$.

7. Parovichnikova E. N. et al. The results of a multicenter randomized trial on the treatment of acute myeloid leukemia of adults // Ter. Arkh. - 2010. - № 82 (7). - P. 5-11.

8. Afanasyev B. V. etal. The experience in non-relative allogeneic transplantation of stem hemopoietic cells in the Clinic of Bone Marrow Transplantation at I. P. Pavlov St-Petersburg Medical Academy // Ter. Arkh. - 2007. - № 79 (7). - P. 36- 43.

9. Bondarenko S. N. et al. Allogeneic hematopoietic stem cell transplantation for acute myeloblastic leukemia in first remission // Ter. Arkh. - 2013. - № 85 (7). - P. 18-25.

10. Suciu $S$. et al. Allogeneic compared with autologous stem cell transplantation in the treatment of patients younger than 46 years with acute myeloid leukemia (AML) in first complete remission (CR1): an intention-to-treat analysis of the EORTC/GIMEMAAML10 trial // Blood. - 2003. - № 102 (4). - P. 1232- 1240.

11. Cornelissen J. J. et al. Results of a HOVON/SAKK donor versus no-donor analysis of myeloablative HLA-identical sibling stem cell transplantation in first remission acute myeloid leukemia in young and middle-aged adults: benefits for whom? // Blood. 2007. - № 109 (9). - P. 3658 - 3666.

12. Cornelissen J. J. et al. The European LeukemiaNet AML Working Party consensus statement on allogeneic HSCT for patients with AML in remission: an integrated-risk adapted approach // Nat. Rev. Clin. Oncol. - 2012. - № 150. - P. 1- 12.

13. de Lima M. et al. Maintenance therapy with low-dose azacitidine after allogeneic hematopoietic stem cell transplantation for recurrent acute myelogenous leukemia or myelodysplastic syndrome: a dose and schedule finding study // Cancer. - 2010. - № 116 (23). - P. 5420 - 5431.

\section{Цитогенетика:}

Таблица 3

Результаты многофакторного анализа ОМА в ПР1

\begin{tabular}{|l|l|l|l} 
& HR & $95 \% \mathrm{CI}$ & $\mathrm{p}$
\end{tabular}

Общая выживаемость

\begin{tabular}{l|l|l|l} 
& 0,37 & $(0,19-0,73)$ & 0,004
\end{tabular}

2,14

2,93

$\begin{array}{ll}(1,24-3,71) & 0,006 \\ (1,45-5,9) & 0,003\end{array}$

\begin{tabular}{r|r}
$(1,45-5,9)$ & 0,003 \\
$(1,0-3,66)$ & 0,05 \\
\hline
\end{tabular}

Бессобытийная выживаемость

\begin{tabular}{|c|c|c|c|}
\hline & 0,39 & $(0,21-0,72)$ & 0,002
\end{tabular}

тный/высокий

,93

$2,07 \quad(1,62-6,0)$

0,008

0,001 Рецияив

\begin{tabular}{|l|l|l|}
0,31 & $(0,15-0,63)$ & 0,001 \\
\hline
\end{tabular}

$\begin{array}{ll}0,47 \quad(0,3-0,74) & 0,001\end{array}$

\begin{tabular}{l}
$1,91 \quad 0,02$ \\
\hline
\end{tabular}

$2,08 \quad(1,14-3,79) \quad 0,02$
3,79

\begin{tabular}{l|l|l}
2,08 & $(1,69-8,51)$ & 0,001
\end{tabular}

$3,32 \quad(1,57-7,0) \quad 0,002$

летальность несвязанная заболеванием

\begin{tabular}{l|c|c|r}
\hline Цитогенетика: & 2,83 & $(1,05-7,6)$ & 0,04 \\
стандартный/высокий & 3,53 & $(1,04-12,0)$ & 0,04 \\
высокий &
\end{tabular}

\section{PEЗЮME}

С. Н. Бондаренко, И. С. Моисеев, И. А. Самородова, Т. Л. Гиндина, М. А. Кучер, О. А. Слесарчук, Л. С. Зубаровскал, Б. В. Афанасьев

Сравнение эффективности химиотерапии и алхогенной трансплантации гемопоэтических стволовых клеток при остром миелобластном лейкозе в первой ремиссии у взрослых

Цель - сравнить эффективность аллогенной трансплантации гемопоэтических стволовых клеток (аллоТГСК) и химиотерапии (ХT) при остром миелобластном лейкозе $(\mathrm{OM} \Lambda)$ в первой ремиссии и выявить факторы, влияющие на результаты лечения. Сравнивалась эффективность в первой ремиссии ОМ $\wedge$ аллоТГСК $(\mathrm{n}=70)$ и ХТ (n=52). Пациенты были разделены по возрасту, уровню лейкоцитов, происхождению ОМ $\Lambda$, цитогенетическим группам риска и ответу на индукционную ХТ. Пятилетняя общая и бессобытийная выживаемость (ОВ и БСВ) были выше в группе аллоТГСК (67 и 65 \% против 46 и $30 \%$ $(\mathrm{p}=0,02$ и $\mathrm{p}=0,001))$. При анализе БСВ от цитогенетических групп риска выявлены преимущества аллоТГСК в стандартной и высокой группах риска (78 \% против $29 \%(\mathrm{p}=0,001)$ и $34 \%$ против $17 \%(\mathrm{p}=0,007))$. Совокупный риск рецидива (РP) составил $24 \%$ у пациентов после аллоТГСК против 57 \% при ХТ (p=0,003). При сравнении РP после аллоТГСК и ХТ от цитогенетических групп риска: стандартная $(\mathrm{HR}, 0,2(\mathrm{CI} 95 \% 0,07-0,56) \quad \mathrm{p}=0,002)$ и высокая $(\mathrm{HR}, 0,27(\mathrm{CI} 95 \% 0,08-0,86) \mathrm{p}=0,03)$. Аополнительными факторами, оказывающими влияние на PP, были: происхождение OM $\lambda$ (de novo) (HR,0,47(CI95 \%0,3 $0,74) \mathrm{p}=0,001)$, гиперлейкоцитоз в дебюте заболевания $(\mathrm{HR}, 1,91(\mathrm{CI} 95 \% 1,09-3,32) \mathrm{p}=0,02)$ и отсутствие ремиссии после одного курса индукции (HR,3,32(CI95 \% 1,57$7,0) \mathrm{p}=0,002)$. Эффрективность аллоТГСК по сравнению с ХТ выше при стандартной и высокой цитогенетических группах риска.

Ключевые слова: острый миелоидный лейкоз, химиотерапия, трансплантация костного мозга. 


\section{SUMMARY}

S. N. Bondarenko, I. S. Moiseev, I. A. Samorodova, T. L. Gindina, M. A. Kucher, O. A. Slesarchuk, L. S. Zubarouskaya, B. V. Afanasyeu

Comparison of the efficacy of chemotherapy and allogeneic hematopoietic stem cell transplantation for acute myeloid leukemia in first remission in adults

The aim of the study was to compare the efficacy of allogeneic hematopoietic stem cell transplantation (alloHSCT) and chemotherapy (CT) of acute myeloid leukemia (AML) in first remission (CR1), to identify factors influencing the results. We compare the efficacy alloHSCT in CR1 $(n=70)$ and CT $(n=52)$. Patients were stratified by age, the level of leucocytes, the origin of AML, cytogenetic risk group and response to induction CT. Five-years overall and disease-free survival (OS and DFS) were higher in the group alloHSCT (67 and $65 \%$ vs 46 and $30 \%$ $(p=0.02$ and $p=0.001))$. Benefits of DFS after alloHSCT was in standard and high-risk cytogenetic groups (78\% versus $29 \%$ $(p=0.001)$, and $34 \%$ vs $17 \%(p=0.007))$. The risk of relapse (RR) was $24 \%$ in patients after alloHSCT vs. $57 \%$ for CT $(p=0.003)$. Comparing the RR after alloHSCT and CT depending on the cytogenetic risk groups: standard (HR0.2(CI95 \%0.070.56) $\mathrm{p}=0.002$ ), and high (HR0.27(CI95\%0.08-0.86) $\mathrm{p}=0.03$ ). Additional factors affect the RR were the origin of AML (de novo) (HR0.47 (CI95 \%0.3-0.74) p=0.001), the hyperleukocytosis (HR1.91 (CI95\%1.09-3.32) p=0.02), and no remission after the first course CT (HR3.32(CI95 \% 1.57-7.0) p=0.002). The efficacy of alloHSCT compared with CT is higher both in standard and high-risk cytogenetic group.

Keywords: acute myeloid leukemia, chemotherapy, allogeneic hematopoietic stem cell transplantation.

\title{
Издательство СПбГМУ
}

\author{
имени академика И. П. Павлова
}

специализируется на издании медицинской, научной

и учебной литературы

Имея в своем составе квалифицированных художественных и научных редакторов, располагая современной полиграфической базой, издательство СПбГМУ может подготовить к печати и издать монографии, брошюры, медицинские журналы, буклеты и другую полиграфическую продукцию, подготовить оригинал-макет любой сложности.

Издательство СПбГМУ принимает заказы на публикацию рекламных объявлений в медицинских журналах и книгах, выпускаемых издательством.

Выпускаемая литература реализуется через магазин и киоск издательства.

Предварительную информацию об условиях оформления, выполнения и оплаты заказов можно получить по адресу:

197089, Санкт-Петербург,

улица Льва Толстого, 6/8

Издательство СПбГМУ имени академика И. П. Павлова 


\section{ПАМЯТКА ДЛЯ АВТОРОВ}

«Ученые записки Санкт-Петербургского государственного медицинского университета имени академика И. П. Павлова» - официальный научный журнал ПСПбГМУ, публикующий статьи по проблемам медицинской науки, практики и преподавания.

Решением Высшей Аттестационной Комиссии (ВАК) Министерства образования и науки РФ журнал «Ученые записки СПбГМУ им. акад. И. П. Павлова» включен в Перечень ведущих рецензируемых научных журналов и изданий, выпускаемых в Российской Федерации, в которых рекомендована публикация основных результатов диссертационных исследований на соискание ученых степеней доктора и кандидата наук.

В журнале имеются следующие разделы:

- передовые статьи;

- оригинальные статьи;

- обзоры и лекции;

- дискуссии;

• в помощь практическому врачу;

- краткие сообщения;

- история и современность;

- исторические даты;

• информация о планах проведения конференций, симпозиумов, съездов;

- реклама

Общими критериями работ, принимаемых для публикации в «Ученых записках СПбГМУ», является актуальность, новизна материала и его ценность в теоретическом и/или прикладном аспектах. Обычной фрормой рукописи является оригинальная статья, текст которой включает в себя введение, материалы и методы исследования, результаты проведенной работы, обсуждение результатов, список литературы.

Обзоры, лекции, статьи по истории медицины и работы, доложенные и одобренные Научным советом СПбГМУ, принимаются после предварительного согласования с Редакцией или по ее заказу.

\section{Правила рецензирования статей:}

1. Редакция обеспечивает экспертную оценку (двойное закрытое рецензирование) материалов, соответствующих ее тематике, с целью их экспертной оценки.

2. Все рецензенты являются признанными специалистами по тематике рецензируемых материалов и имеют в течение последних 3 лет публикации по тематике рецензируемой статьи.

3. Один из рецензентов является членом редколлегии журнала

4. Редакция обеспечивает экспертную оценку (двойное закрытое рецензирование) рукописей.

5. На основании письменных рецензий и заключения Редколлегии рукопись принимается к печати, высылается автору (соавторам) на доработку или отклоняется.

6. В случае отказа в публиковании статьи редакция направляет автору мотивированный отказ

7. Редакция обязуется направлять копии рецензий в Министерство образования и науки Российской Федерации при поступлении в редакцию издания соответствующего запроса

8. Рецензии хранятся в издательстве и в редакции издания в течение 5 лет.

9. Статьи, посвященные диссертационным исследованиям, публикуются в журнале бесплатно.

\section{Аокументы, необходимые для представления статьи в редакцию:}

1) статья на бумажном носителе в 2-х экземплярах с подписями всех авторов статьи с указанием их должностей и званий, рабочих адресов и телефонов для связи, а также с подписью руководителя подразделения;

2) электронный вариант статьи на CD-диске, соответствующий бумажному варианту;

3) направление от учреждения для публикации статьи в журнале «Ученые записки СПбГМУ»;

4) экспертное заключение руководителя об отсутствии в материале статьи сведений, не подлежащих опубликованию, подтверждением, что данный материал не был опубликован в других изданиях и не принят к печати другим издательством/издающей организацией, сообщением о возможном конфликте интересов;

5) письмо-сопровождение, подтверждающее передачу прав на публикацию статьи в неограниченном количестве экземпляров, с подписями всех авторов. 
Электронные версии статей, полученные через Интернет, без оригиналов указанных вышедокументов редакцией не рассматриваются.

Редакция оставляет за собой право публиковать принятые к печати статьи в том виде и в такой последовательности, которые представляются оптимальными для журнала.

\section{ЮРИДИЧЕСКИЕ ТРЕБОВАНИЯ}

Подача рукописи означает, что описываемая работа не публиковалась ранее; что она не находится на рассмотрении где-либо еще; что ее публикация одобрена всеми авторами (соавторами) и организацией, где была выполнена работа.

Автор (соавторы) предоставляют издателю на срок до 10 лет следующие права:

- право на воспроизведение работы без ограничения тиража экземпляров;

- право на опубликование, обнародование, дублирование, тиражирование или иное размножение произведения;

- право на распространение произведения любым способом, в том числе через Интернет;

- право на публичное использование и демонстрацию произведения в информационных, рекламных и прочих целях;

- право на доведение до всеобщего сведения;

- право на переработку произведения и внесение изменений;

- право на использование метаданных произведения (название, имя автора (правообладателя), аннотация, библиографические материалы и пр.) путем распространения и доведения до всеобщего сведения, обработки и систематизации, а также включения в различные базы данных и информационные системы;

- право переуступить на договорных условиях частично или полностью полученные по настоящим правилам публикации права третьим лицам без выплаты автору (соавторам) вознаграждения.

Поступление статьи в редакцию подтверждает полное согласие автора (соавторов) с правилами журнала.

\section{ОФОРМЛЕНИЕ РУКОПИСИ}

Статьи представляются в редакцию на дисках (CD-диск), подготовленных на любом IВМ-совместимом компьютере в текстовом редакторе «Word» (версия 7.0 и выше) с распечаткой текста на белой бумаге в двух экземплярах. Работы должны быть напечатаны шрифтом TimesNewRoman, 14 кеглем, через два интервала с полями сверху - 20 мм, слева - 30 мм, справа -10 мм, снизу - 20 мм. Все страницы статьи должны быть пронумерованы арабскими цифрами.

Таблицы. Каждая таблица должна быть напечатана на отдельной странице, иметь номер и название. Все графы в таблицедолжны иметь заголовок, сокращения слов в таблице допускаются только в соответствии с требованиями ГОСТ 1-5-68. Ранее опубликованный материал указывается в форме ссылки в конце заголовка таблицы. При внесении в материал таблиц изменений, необходимо предоставить письменное разрешение на воспроизведение от их автора (владельца).

Рисунки должны быть выполнены в двух экземплярах на одной стороне отдельных листов плотной белой гладкой бумаги или ватмана, размером не более $20 \times 30$ см, черной тушью; микрофротографии и рентгенограммы - на глянцевой бумаге (холодный глянец). Размер фротографий - 9× 12 см. На обратной стороне каждого рисунка или фото указываются ФИО первого автора, название статьи, номер рисунка и отмечается верх и низ. На рисунке должно быть минимальное количество обозначений, все пояснения выносятся в подрисуночные подписи. Аля всех иллюстративных материалов в тексте указывается их место. Аля иллюстраций (рисунков, схем, диаграмм, фротографий), имеющих электронную версию, необходимо предоставлять ее в виде отдельного файла в форматах tif, рсх, bmp, xls и т. п. на CD-диске. Аля ранее опубликованных иллюстраций необходимо указать оригинальный источник в форме ссылки в конце подписи. При внесении в материал иллюстраций изменений, необходимо предоставить письменное разрешение на воспроизведение от их автора (владельца). Люди, изображенные на фотографиях, не должны быть узнаваемыми, либо автор должен представить в редакцию письменное разрешение на публикацию этих иллюстраций от лица, изображенного на фотографии.

Аля оригинальной статьи суммарный объем (текст, иллюстрации, список литературы, резюме на русском и английском языках и ключевые слова) не должен превышать 10 страниц (бумага А4), напеча- 
танных 14 кеглем, через 2 интервала. Краткое сообщение (до 4-х страниц) оформляется аналогичным образом, число иллюстраций и таблиц - не более трех, список использованной литературы в краткое сообщение не включается, резюме не требуется.

Объем и оформление других видов работ (обзоры, лекции или иное) согласуются с Редакцией заранее.

\section{СТАТЬИ, НАПРАВЛЕННЫЕ В ЖУРНАЛ, ДОЛЖНЫ ИМЕТЬ}

Титульный лист (печатается на отдельной странице) включает: 1) название статьи; 2) ФИО автора (соавторов); 3) ученую степень автора (соавторов); 4) место (места) выполнения работы, служебный адрес (адреса) и телефон; 5) фразу «Авторы ознакомлены с памяткой для авторов и полностью согласны с имеющимися требованиями»; 6) подпись автора (соавторов); 7) источники финансирования. В нижней части этого листа следует проставить должность, ученое звание, степень, а также телефон, факс и е-таil (если имеются) автора, с которым Редакция будет поддерживать связь.

Аанные об авторах указываются в последовательности, которая определяется их совместным решением и подтверждается подписями на титульном листе. Иные лица, внесшие вклад в выполнение работы, недостаточный для признания авторства (не могущие принять на себя ответственность за содержание работы, но оказавшие техническую, финансовую, интеллектуальную помощь), должны быть перечислены (с их письменного разрешения) в разделе «Выражение признательности» после текста статьи.

Списоклитературы (печатается с новой страницы) составляется в алфавитном порядке: сначала работы отечественных авторов, затем иностранных авторов. Работы отечественных авторов, опубликованные на иностранных языках, помещаются среди работ иностранных авторов, а работы иностранных авторов, опубликованные на русском языке, - среди работ отечественных авторов. В тексте статьи ссылки нумеруются в квадратных скобках: [1], [3-6], [8, 9].

Фамилии иностранных авторов, упоминаемые в тексте статьи, даются в оригинальной транскрипции.

Резюме, объемом не более 150 слов, в котором коротко, но исчерпывающе излагается основное содержание работы. Резюме должно включать ФИО авторов, название работы и место ее выполнения; ключевые слова (не более 6), способствующие индексированию статьи в информационно-поисковых системах. Вся указанная информация приводится на русском и английском языках.

\section{ЭТИЧЕСКИЕ СТАНДАРТЫ}

Защита прав человека и животных. Рукописи, подаваемые на публикацию, должны содержать заявление о том, что исследования с участием человека/людей одобрены соответствующим комитетом по этике и проводились в соответствии с законодательством.

Соблюgение прав пациентов и конфияенциальность. Пациенты имеют право на соблюдение конфиденциальности, которую нельзя раскрывать без их согласия. Информация, позволяющая установить личность, включая ФИО пациентов, номера больниц и историй болезней, не должна публиковаться в виде письменных описаний и фотографий без предоставления письменного согласия пациента или его законного представителя. Авторы статьи должны предоставить в Редакцию письменное информированное согласие пациента или его законного представителя на распространение информации и сообщить об этом в статье.

\section{PA3HOE}

1. В статью рекомендовано включать не более 5 соавторов.

2. Титульный лист в общий объем оригинальной статьи или краткого сообщения не включается.

3. В материалах, направленных в журнал, должна быть использована система СИ, за исключением размерности величин, традиционно измеряемых в других мерах.

4. Все сокращения и аббревиатуры, используемые в статье, должны быть расшифрованы в тексте при их первом упоминании, кроме символов химических элементов и сокращенных названий метрических единиц.

5. Исправленные автором после рецензирования и перепечатанные рукописи возвращаются в редакцию не позднее одного месяца, а исправленные гранки - через одну неделю.

6. Авторский гонорар и оплата труда по рецензированию рукописей не предусмотрены.

7. Материалы, не принятые к печати, возвращаются авторам по их заявлению по месту нахождения Редакции журнала не позднее, чем в течение трех месяцев с момента отказа в печати. 
8. Один авторский экземпляр журнала можно получить в Издательстве СПбГМУ им. акад. И. П. Павлова. Электронная версия журнала размещается в Интернете по адресу адресу http://1spbgmu.ru/ru/. Pacсылка авторских экземпляров журнала почтой не осуществляется.

\section{ОФОРМЛЕННЫЕ В СООТВЕТСТВИИ С НАСТОЯЩИМИ ПРАВИЛАМИ РУКОПИСИ СЛЕДУЕТ НАПРАВЛЯТЬ ПО АДРЕСУ:}

197022, Санкт-Петербург, ул. ^. Толстого, 6/8, Первый Санкт-Петербургский государственный медицинский университет им. акад. И. П. Павлова, Редакция журнала «Ученые записки СПбГМУ». телефон: 338-66-77, 338-70-07

фракс: 8 (812) 338-66-77

e-mail:nauka@spb-gmu.ru

www.1spbgmu.ru/ru/

Главный редактор - академик РАН, профессор С. Ф. Багненко

Зам. главного редактора - профессор Э. Э. Звартау

Зам. главного редактора - чл.-корр. РАН, профрессор Ю. С. Полушин

\section{ВНИМАНИЮ ЧНТАТЕЛЕЙ!}

Сообщаем Вам, что на журнал «Ученые записки» проводится подписка по каталогу «Пресса России». Подписной индекс для организаций и частных лиц - 29248.

Информацию о подписке на журнал «Ученые записки» Вы также можете получить в издательстве СПбГМУ им. акад. И. П. Павлова.

Agpec: 193089, Санкт-Петербург, ул. ^. Толстого, 6/8

Телефон: (812) 338-66-77

Факс: (812) 338-66-77 


\section{REGULATIONS FOR AUTHORS}

The «Record of the I. P. Pavlov St. Petersburg State Medical University» («Record of IPP-SPSMU») is the official journal of the IPP-SPSMU. It publishes reports on the problems of medical science, practical work and teaching.

In accordance with the resolution of the Supreme Attestation Comission (SAC) of the Ministry of Education and Science the journal «Record of the I. P. Pavlov St. Petersburg State Medical University» is included in the list of the leading reviewed scientific journals issued in the Russian Federation and is recommended for publication of the main results of dissertation researches for scientific degree of a Candidat of Science and of a Doctor of Science.

The journal offers the fillowing sections:

- editorials;

- origival papers;

- reviews and lectures;

- discussions;

- to assist the practitioner

- brief information;

- hystory and present day events;

- historical calendar;

- information on the schedule of conferences, symposia, and congresses;

- advertisement.

The general criteria for publication of a manuscript submitted to the "Record of IPP-SPSMU» are novelty and actuality of the presented material, its theoretical and practical value. The accepted form of a manuscript is the original text incorporating introduction, materials and methods, results, discussion, and summary (in Russian and English).

Reviews, lectures, and papers approved by the Scientific Board are admitted for publication after the Editorial Board approval.

\section{Rules of the articles reviewing:}

1. The Editorial board provides practitioner led assessment (double-blind review) of the materials according to their category for the expert assessment.

2. All reviewers are the acknowledged experts on the subject of the reviewed materials and have publications on the subject of the reviewed article during the past 3 years.

3. One of the reviewers is a member of the Editorial board.

4. The Editorial board provides practitioner led assessment (double-blind review) of the manuscripts.

5. Based on written reviews and the conclusion of the Editorial board of the manuscript is accepted for publication, sent to the author (co-authors) for revision, or it is rejected.

6. In case of refusal in publication of article, the Editorial board sends a reasoned refusal to the author.

7. The Editorial board is obliged to send copies of reviews to the Ministry of Education and Science of the Russian Federation upon receipt of the corresponding request at the editorial office.

8. The reviews are kept at the publishing house and the editorial office for 5 years.

9. The articles devoted to the dissertation research are published in the journal free of charge.

\section{The documents to be presented to the Editorial Board are:}

1) the article on the paper sheet in two copies with signatures of all co-authors, their posts and ranks, office telephones for communication, and the department chief signature;

2) an electron variant of the article on a CD-disc;

3) recommendation from the office for publication of the paper in the journal «Record of IPP SPSMU»;

4) an expert conclusion from the office supervisor on the absence of any information not to be published; on confirmation of the fact that the material presented had not been published in other journals nor has been accepted by other publishing organizations; as well as information on the possible conflict of interests;

5) a covering letter confirming transfer of the rights on publication of the article in unlimited number of copies signed by all authors.

The electron versions of the articles without the above mentioned documents are not accepted. 
The Editorial Board is authorized to publish the accepted manuscripts in the most optimal manner for the Journal.

\section{LEGAL REQUIREMENTS}

Submission of a manuscript for publication signifies that the work had not been published anywhere; that it had not been accepted by any other publishing house; that its publication was approved by the authors and by the institution where the work had been done.

The author/co-authors grant the Publishing House - for ten years - the following rights:

- to reprint the work without restriction of the copies;

- to publish, promulgate and duplicate the work;

- to distribute the work by any method, including Internet;

- to openly use and demonstrate the work for information and advertisement;

- to make corrections and to recast the text;

- to use the metadata (the title of the work, the author`s name, annotation, bibliographic material etc.) for distribution and promulagation, for correction and systematization, for including the information into various data bases and other information systems;

- to cede the information as a whole or partially - in accordance with the rules currently in force - to a third person (royalties are not provided).

Submission of a manuscript to the Editorial Board is a confirmation of the author(s) agreement with the requirements.

\section{MANUSCRIPT PREPARATION}

A manuscript of a Regular Article should be printed in triplicate, double-spaced using $2.5 \mathrm{~cm}$ wide margins all around, and restricted to $10 \mathrm{~A} 4$ numbered pages. The space allotted should incorporate all sections of the manuscript, including the numbered, alphabetically arranged, and full (with titles in quotation) List of References. In the text, the references are given as figures in the square parentheses. Figures and/or photos are submitted on the separate sheets, with indication of their optimal place on the left margins of the text. Up to six single $(9 \times 12$ $\mathrm{cm})$ or two composite $(14 \times 18 \mathrm{~cm})$ halftone photos ready for PC-scanning are accepted, each one with a scale bar, markings (if necessary), and figure legends as well as magnifications and staining modes on a separate sheet. All illustrations should be identified on the back with figure number, running title of the paper, name of the first author, and an arrow indicating the top. Short Communication should not exceed four pages, with three single or one composite photos, ten quotations, and the arrangement as in a Regular Article. Style and length for the Review Articles, Lectures, and History of Medicine Articles are to be stipulated with the Editor before the manuscript submission.

Manuscripts not conforming to the Regulations will be returned to the authors without assessment.

Title page. The title page bears the name(s) of the author(s), the title of the manuscript, the institution(s) where the work was done, all addresses and signatures of the authors. On the bottom of the Title Page, a full name, person's duties, academic degree(s), affiliation, as well as the address, phone, facsimile, and e-mail coordinates are placed as to the author to whom the Journal should communicate.

Summary. Summary should written (150 words or less) on a separate sheet and followed by up to six key words, in italics, on a separate line.

Disk Submission. The CD-disk file of all sections of the paper, except for photos, should accompany the manuscript prepared on PC-IBM (not Apple Mackintosh) compatible computer. Include an ASCII version on the disc, together with the word-processed version.

\section{ETHICS STANDARDS}

Defence of the rights of people and animals. A manuscript to be submitted shold include a statement that the investigation of the problems concerning human beings and animals had been approved by a corresponding Commission on Ethics and had been carried out in full accordance with the legislation.

Observation of the patient's rights and confidence. A patient has a right to confidence that cannot be violated without his/her consent. The data on the patients name, the hospital number, and the case history are not to be disclosed by written description and photos without written consent of the patient or his legal representative. The author of a manuscript should produce a written consent of the patient or his/her legal representative to spreading of the data and mention the fact in the manuscript. 


\section{MISCELLANEOUS}

1. The namber of the authors showld not exceed five persons.

2. Title page, summary, any figures, and figure legends are not included in the space allotted to a manuscript, of either Regular Article or Short Communication type.

3. All measurements should be presented in the symbols of the Numerical System except the values with specific notations.

4. All abridgements and abbreviations - except the chemical element symbols and the metric system units should be deciphered when first mentioned in the text.

5. It is advisable for the contributors to return the revised and re-printed versions of the manuscripts, and also the approved galley proofs within one months and one weeks after receipt, respectively. Otherwise, publishing of the manuscripts can be delayed.

6. The author and the reviewer royalties for the papers revised and published are not provided for.

7. A manuscripts rejected by the IPP SPSMU may be returned to the authors at the Editorial Board office on receiving a request within 3 months.

8. The author's copy of the journal may be obtained in the Editorial office. The electron version of the Record os given in Internet the address is http://www.spb-gmu.ru. Delivery of the Journal to the authors is not provided.

\section{SUBMISSION ADDRESS:}

197022, St. Petersburg, 6-8 Lev Tolstoy str., the I. P. Pavlov St. Petersburg State Medical University

Editorial Office of the journal «Records of SPbSMU»
Tel.: 7 (812) 338-66-77, 338-70-07

Fax: 7 (812) 338-66-77

e-mail: nauka@spb-gmu.ru www.1spbgmu.ru/ru/

Editor-in-chief - S. F. Bagnenko, MD, PhD, DMSc, academician of RAMS, professor

Deputy Editors - E. E. Zvartau, MD, PhD, DMSc, professor

Deputy Editors - Yu. S. Polushin, MD, PhD, DMSc, professor, corresponding member of RAS 
Обложка и художественное оформление E. В. Корнилова

Компьютерная верстка и подготовка оригинал-макета А. А. Чиркова

Корректор B. А. Черникова

Журнал зарегистрирован

Государственным комитетом Российской Федерации по печати.

Свидетельство № 017631 от 22 мая 1998 г.

Подписано в печать 28.09.2015. Формат бумаги $60 \times 90^{1} \%$.

Бумага офсетная. Печать офсетная. Печ. ^. 10. Тираж 1000 экз. № 937/15.

Издательство СПбГМУ им. акад. И. П. Павлова, 197089, Санкт-Петербург, ул. Аьва Толстого, 6/8.

(C) уЧЕНЫЕ ЗАПИСКИ СПбГМУ им. акад. И. П. Павлова, 2015 